\title{
ACOMPANHAMENTO E CONDUTA DA MÁ ROTAÇÃO INTESTINAL NO ADULTO: RELATO DE CASO
}

\section{FOLLOW-UP AND MANAGEMENT OF ADULT INTESTINAL MALROTATION: CASE REPORT}

\section{Autores \\ Isabella Victória Pereira De Barros ${ }^{1}$ Isabella Carvalho Pagnussat ${ }^{1}$ Laíse de Paula Maitelli ${ }^{1}$ Eduardo Gidrão Dantas² Talita Luz Garcia ${ }^{2}$ Emanuel Gonçalo Leite De Almeida ${ }^{3}$ Fernanda Vanessa Pereira De Barros ${ }^{4}$ Mário Renato da Silva \\ 1. Acadêmicas de Medicina do Centro Universitário de Várzea Grande; \\ 2. Médicos residentes em Cirurgia Geral pelo Hospital Santa Rosa; \\ 3. Médico graduado pela Universidade de Cuiabá, residência médica em Cirurgia Geral pelo Hospital Santa Rosa, Cirurgião do Aparelho Digestivo; \\ 4. Médica graduada pela Universidade de Cuiabá, residência médica em Cirurgia Geral no Hospital Santa Rosa e Coloproctologia no Hospital Alberto Rassi. \\ 5. Médico graduado pela Universidade Federal do Mato Grosso, residência médica em Radiologia e Diagnóstico por Imagem pela Universidade de Brasília.}

\section{RESUMO}

Objetivo: Respectivo estudo em relato de caso acerca da má rotação intestinal em paciente adulto sintomático, portador de síndrome heterotáxica, possui como ênfase relatar com detalhamento o caso com seguimento e conduta. Método: Análise retrospectiva do prontuário do paciente, com formulário de autorização, cedido pela Residência de Cirurgia Geral do Hospital Santa Rosa no Município de Cuiabá - Mato Grosso. Descrição do Caso: Homem negro, 51 anos, internado com quadro de abdome agudo obstrutivo, através do auxílio de exame de imagem e da laparotomia com diagnóstico de má rotação intestinal, reportamos o pós-operatório e conduta mediante o caso. Discussão: As anomalias da rotação intestinal são descritas como falhas parciais ou completas no desenvolvimento normal do intestino. Por ser congênita, $90 \%$ dos diagnósticos são realizados ainda no primeiro ano de vida, apresentação em adultos é rara, pode ser assintomática ou apresentar quadros obstrutivos e, assim como outras anomalias, também pode ser justificada pela síndrome heterotáxica.

Palavras-chave: Derivação Ventrículo Peritoneal; Complicações Abdominais; Extrusão Anal.

\section{ABSTRACT}

Objective: The respective study in a case report about intestinal malrotation in a symptomatic adult patient with heterotaxic syndrome, emphasizes reporting the case in detail with follow-up and management. Method: Retrospective analysis of the patient's medical record, with authorization form, provided by the General Surgery Residency of Hospital Santa Rosa in the city of Cuiabá - Mato Grosso. Case Description: Black man, 51 years old, hospitalized with acute obstructive abdomen, through the aid of image examination and 
1. Araújo URMF, et col. Má rotação intestinal em adulto, relato de caso e revisão da

literatura. ABCD. Arq Bras Cir Dig. 2009; 22(4): 240-242. [Citado em 20 Mar 2021] Disponível em: https://www.scielo.br/pdf/abcd/v22n4/ v22n4a13.pdf

3. Fonseca MK, el col. Má rotação intestinal em adulto. Relatos Casos Cir. 2019; 5(3): e2292. [Citado em 20 Mar 2021] Disponível em: https://cdn.publisher.gn1.link/

relatosdocbc.org.br/pdf/v5n3a17.pdf

8. Meneghetti GC, et col. Síndrome

heterotáxica: relato de caso. São Paulo. Radio Bras. 2014; 47(1): 54-56. [Citado em 20 Mar

2021] Disponível em: https://www.scielo.br/ pdf/rb/v47n1/pt_0100-3984-rb-47-01-54.pdf laparotomy with diagnosis of intestinal malrotation verified, we report the postoperative and conduct according to the case. Discussion: Anomalies of intestinal rotation are described as partial or complete failures in the normal development of the intestine. As it is congenital, $90 \%$ of diagnoses are made in the first year of life, presentation in adults is rare, this could be asymptomatic or present obstructive conditions and could be justified by heterotaxic syndrome.

Keywords: Congenital Abnormalities; Embryology; Colorectal Surgery.

\section{INTRODUÇÃO}

A má rotação intestinal é uma anomalia congênita, decorrente da não rotação ou rotação incompleta do intestino médio, durante o período embrionário. ${ }^{1}$

Caracteriza-se por defeitos nas etapas de herniação, rotação e fixação do intestino primitivo, que resultam em alterações anatômicas, tais como a junção duodeno-jejunal localizada no quadrante superior direito e o ceco no abdome superior. Como resultado, tem-se uma base mesentérica estreita, o que predispõe ao volvo intestinal. ${ }^{2}$

De modo típico, manifesta-se nos primeiros meses de vida, com evidências clínicas em $64 \%$ dos casos e, com $90 \%$ dos diagnósticos realizados até o primeiro ano de vida.

A incidência desta malformação permanece estimada em 1:200 a 1:6000 dos nascidos vivos. A condição em adultos é rara, com incidência de $0,2 \%$, sendo que $15 \%$ dos pacientes permanecem assintomáticos durante toda a vida.

Além disso, assim como outras alterações, pode ser justificada pela síndrome heterotáxica, na qual o paciente apresenta um arranjo anormal de órgãos, vasos e, também, dimorfismos. ${ }^{1,3,8}$

Por se tratar de uma condição incomum em idade tardia, o diagnóstico frequentemente é difícil de ser definido nos casos sintomáticos. Nesse sentido, o presente trabalho relata um caso de má rotação intestinal em paciente adulto sintomático, com ênfase no seu seguimento e conduta. 


\section{MÉTODOS}

Este trabalho teve como base, uma análise retrospectiva do prontuário da paciente J.S.S.M. 0 formulário de autorização foi assinado pelo paciente e por todos os integrantes da pesquisa, médicos e acadêmicas, que conduziram o caso.

O estudo foi realizado em novembro de 2020 e cedido pela Residência de Cirurgia Geral do Hospital Santa Rosa no Município de Cuiabá - Mato Grosso, sendo o relato de continuidade no Hospital e Maternidade Santa Helena, onde foram tomadas as condutas iniciais até a alta da paciente. O único critério utilizado foi inclusivo, com finalidade do direcionamento do relato de caso, conforme o tema dissertado.

\section{DESCRIÇÃO DO CASO}

Paciente J.S.S.M, sexo masculino, negro, 51 anos, foi internado na enfermaria do Hospital Beneficente Santa Helena no Município de Cuiabá - MT em novembro de 2020. No momento, referia parada de eliminação de flatos e fezes há 4 dias.

Junto a isso, tinha queixas de náuseas e vômitos (4 episódios diários), além de dor abdominal tipo cólica intensa e discreta distensão do abdome. Ao exame físico, paciente apresentava-se em regular estado geral, afebril, desidratado +/4+, acianótico, anictérico e com tempo de perfusão capilar preservado.

No exame específico do abdome constatou-se uma discreta distensão e ruídos hidroaéreos ausentes. Dessa forma, levantou-se a hipótese diagnóstica de Abdome Agudo Obstrutivo.

Ao resultado dos exames laboratoriais observamos os seguintes resultados: hemácias de 5,3 milhões/mm3; hemoglobina de $14,3 \mathrm{~g} / \mathrm{dL}$; hematócrito de 41\%; leucograma, eletrólitos e coagulograma sem alterações; glicemia de jejum de 198 mg/dL (mg/dL); uréia de 183 mg/dL; creatinina de $2,12 \mathrm{mg} / \mathrm{dL}$.

Dessa forma, como conduta foi realizada ressuscitação volêmica e descompressão intestinal com passagem de sonda nasogástrica, e solicitado tomografia computadorizada de abdome total sem contraste endovenoso devido a anormalidade da função renal (Figura 1; Figura 2; Figura 3), além de uma laparotomia exploratória. 
Figura 1 - Corte tomográfico do abdome no plano coronal sem contraste endovenoso mostrando a disposição das alças de intestino grosso somente à direita da cavidade abdominal nas setas brancas. Nota-se também dilatação difusa de alças de intestino delgado indicando sinais de obstrução.

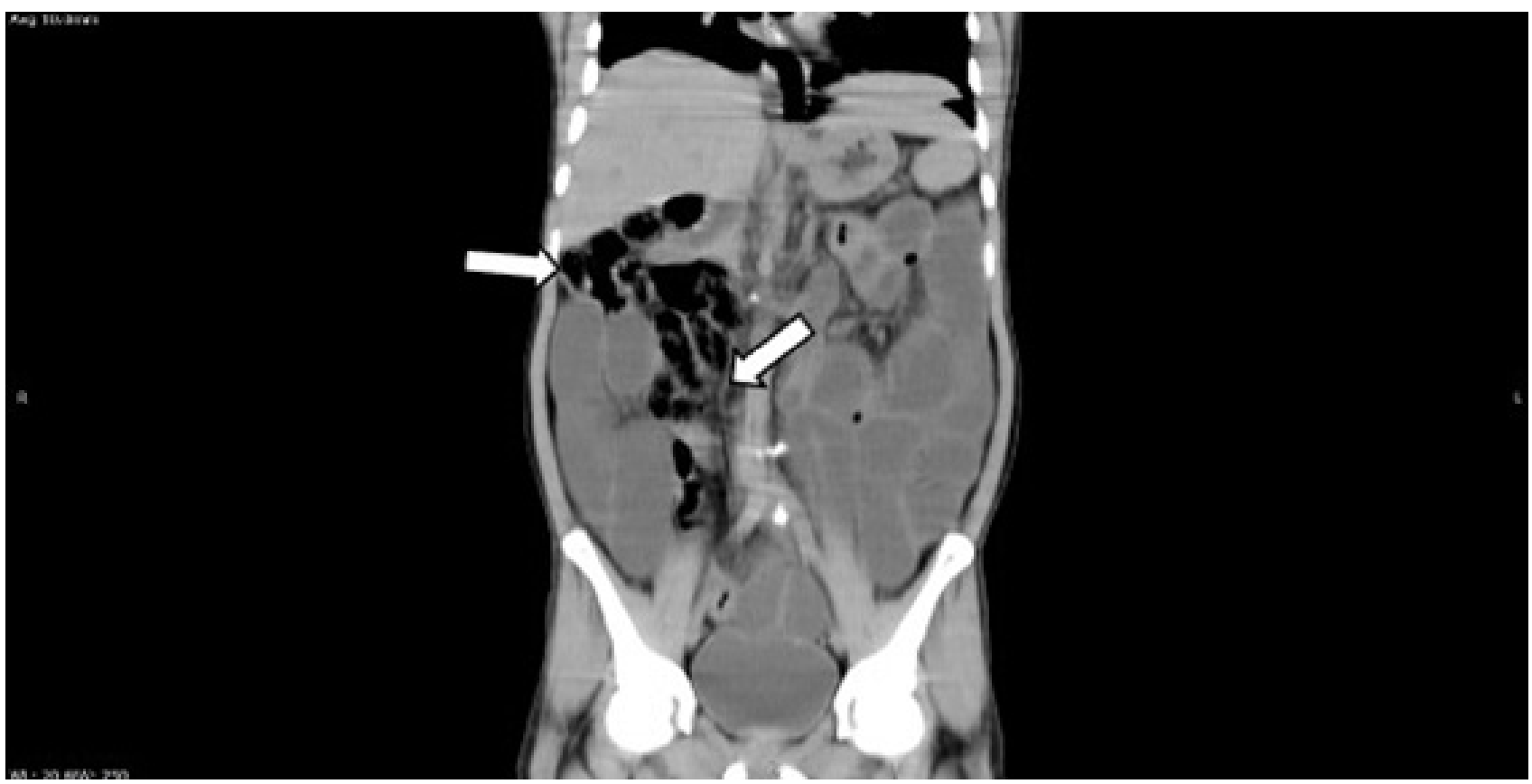

Figura 2 - Corte tomográfico do abdome no plano coronal sem contraste endovenoso mostrando torção dos vasos mesentéricos (seta), indicando a possibilidade de volvo.

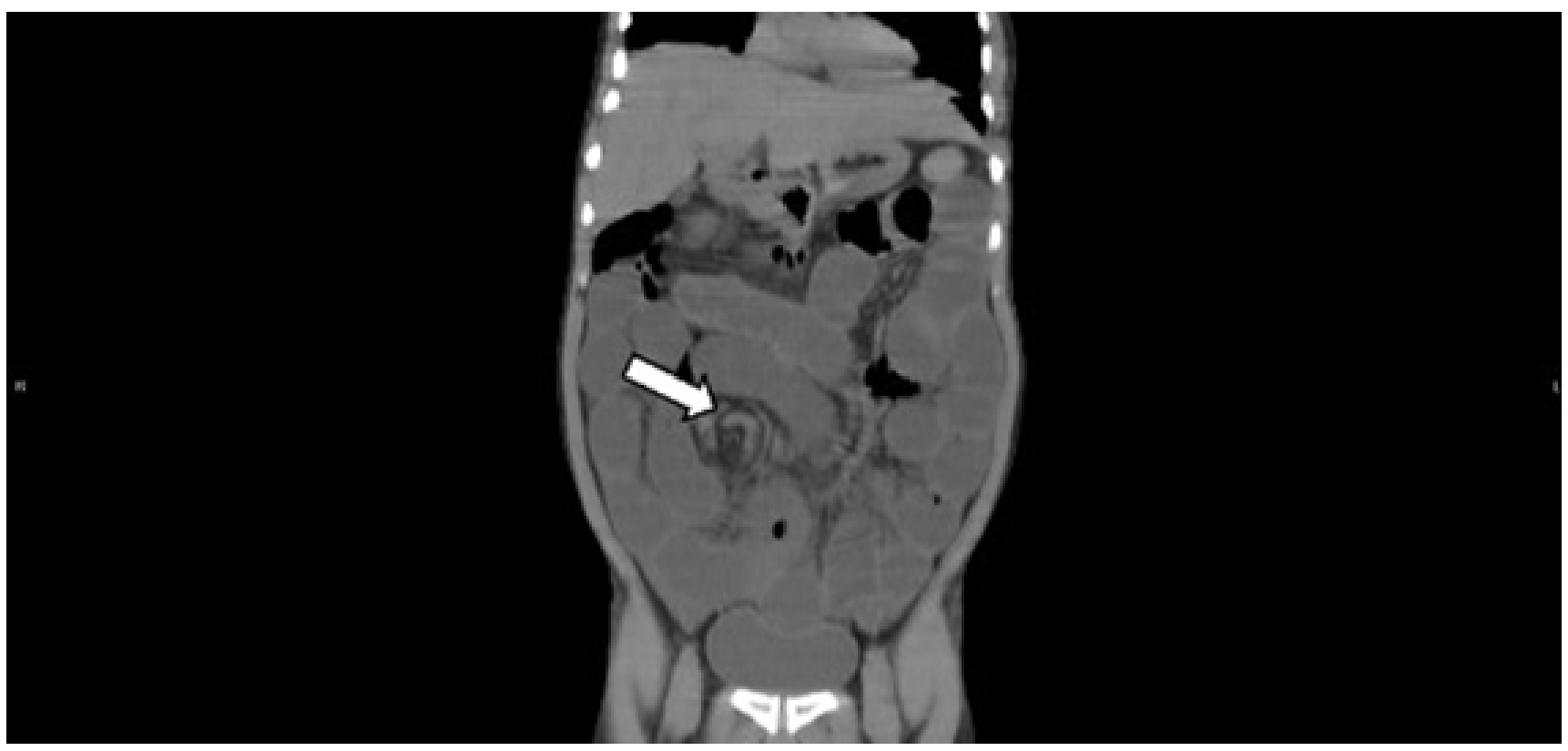


Figura 3 - Corte tomográfico do abdome no plano axial sem contraste endovenoso do paciente com má rotação intestinal e heterotaxia mostrando poliesplenia e pâncreas com dimensões reduzidas. Setas brancas os pequenos baços e cabeça da seta branca o pâncreas.

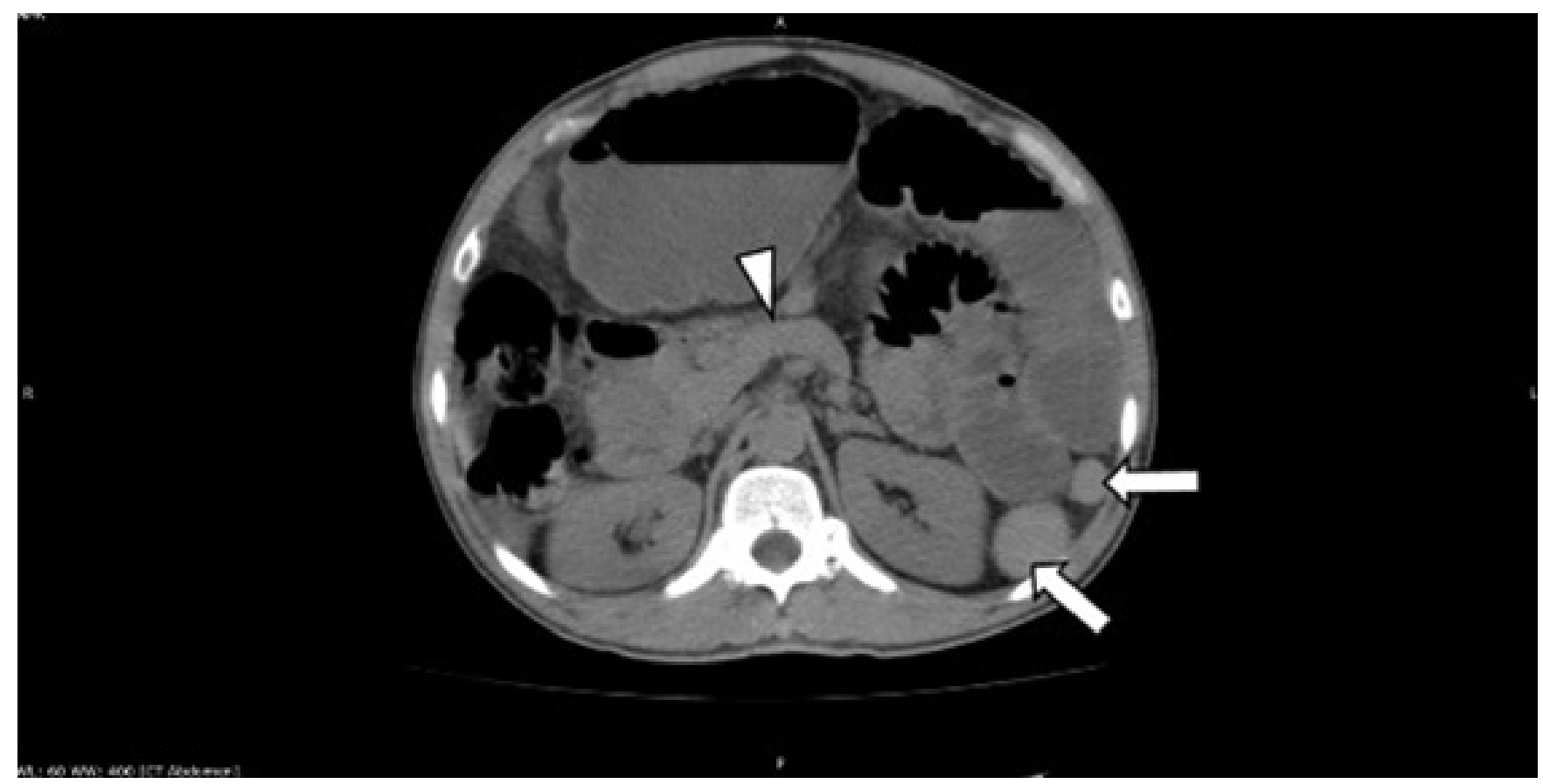

No mesmo dia, foi realizada a laparotomia exploradora, a partir de uma incisão mediana. A partir disso, foi identificado: dilatação de alças intestinais difusamente; ausência de líquido livre em cavidade abdominal; volvo de sigmoide; região cecal dilatada e com pontos de isquemia intestinal; cólon descendente, sigmoide e ceco localizados em região de ângulo hepático; ausência de fixação do ceco em região retroperitoneal e Divertículo de Meckel há 40 centímetros da válvula ileocecal. A partir disso, a equipe cirúrgica tomou as seguintes condutas: colectomia total com uso de grampeador linear número 80, mantendo o reto alto, enterectomia de aproximadamente 45 centímetros do íleo e a 40 centímetros da válvula ileocecal com ressecção do Divertículo de Meckel. Ainda foram feitas lavagem intra-operatória exaustiva da cavidade abdominal com soro fisiológico aquecido, revisão vigorosa da hemostasia, realização e maturação de ileostomia terminal em flanco direito, contagem de compressas, fechamento da aponeurose com fio Nylon 0.0 laçado, síntese da pele com Nylon 3.0, curativo oclusivo na ferida operatória e envio de material para anatomopatológico.

Após a cirurgia o paciente foi encaminhado aos cuidados intensivos em unidade de terapia intensiva, com dieta zero, analgesia de horário, antibioticoterapia com Ciprofloxacino de 400 miligramas a cada doze horas por via endovenosa e Metronidazol de 250 miligramas a cada 8 horas e dois comprimidos por vi oral.Ademais, prescritos medicamentos para prevenção de Tromboembolismo Pulmonar e Trombose Venosa Profunda, além de controle glicêmico.

Em unidade de terapia intensiva, paciente apresentou melhora dos níveis de creatinina e uréia. 
1. Araújo URMF, et col. Má rotação intestinal em adulto, relato de caso e revisão da literatura. ABCD. Arq Bras Cir Dig. 2009; 22(4) 240-242. [Citado em 20 Mar 2021] Disponível em: https://www.scielo.br/pdf/abcd/v22n4/ v22n4a13.pdf

4. Moore KL, Persaud V. Embriologia Básica. 9a edição. Rio de Janeiro: Guanabara. Elsevier. 2004. Capítulo 13. Sistema Digestivo; $p$ 222-250
Em avaliação da Nefrologia foi relatada Lesão Renal Aguda pré-renal, com orientações de utilização de heparina não fracionada e expansão volêmica. Ao segundo dia de pós-operatório, o paciente apresentou-se em bom estado geral; afebril; respirava em ar ambiente; sem uso de drogas vasoativas; havia boa aceitação de dieta pastosa e ileostomia funcionante. Ao terceiro dia, o paciente tinha uma boa aceitação de dieta branda; a ferida operatória estava em bom aspecto, sem saída de secreções ou sinais flogísticos. Havia, ainda, um controle rigoroso da glicemia com uso de Insulina NPH e Insulina Regular. Ao quarto dia de pós-operatório paciente recebeu alta da unidade de terapia intensiva e após um dia em enfermaria sem intercorrências, recebeu alta hospitalar, com acompanhamento semanal em ambulatório.

\section{DISCUSSÃO}

A formação do intestino primitivo ocorre durante a quarta semana embrionária e se divide, de modo descritivo, em três partes: intestino anterior, médio e posterior. Cada região recebe suprimento de suas respectivas artérias: artéria celíaca, artéria mesentérica superior e artéria mesentérica inferior. ${ }^{4}$ Como característica do desenvolvimento, o intestino médio se alonga e produz uma herniação fisiológica, através do ducto vitelínico, com rotação de $90^{\circ}$ no sentido anti-horário, em torno do eixo da artéria mesentérica superior. Durante a décima semana, inicia-se o processo de redução da hérnia fisiológica do intestino delgado, com rotação adicional anti-horária de $180^{\circ}$ no eixo vascular mesentérico. À medida que o intestino adota suas posições finais, ocorre a fixação anatômica do mesentério à parede abdominal. ${ }^{3}$

As anomalias da rotação intestinal foram descritas por Dott ${ }^{5}$, em 1923, e ocorrem por falhas parciais ou completas no desenvolvimento normal do intestino. Por ser congênita, 90\% dos diagnósticos são realizados ainda no primeiro ano de vida, como resultado da investigação de dor abdominal e vômitos biliosos em neonatos. A apresentação em adultos é rara, com incidência de $0,2 \%$, descrita na literatura, de forma intermitente, nas últimas quatro décadas.6 Segundo Araújo e El Tawil ${ }^{1}$, a maioria dos casos de má rotação intestinal em adultos é assintomática e, dentre aqueles que manifestarão sintomas, a evolução pode ser aguda ou crônica. A forma aguda cursa com quadros de obstrução intestinal alta, manifesto por 
1. Araújo URMF, et col. Má rotação intestinal em adulto, relato de caso e revisão da literatura. ABCD. Arq Bras Cir Dig. 2009; 22(4): 240-242. [Citado em 20 Mar 2021] Disponível em: https://www.scielo.br/pdf/abcd/v22n4/ v22n4a13.pdf

3. Fonseca MK, el col. Má rotação intestinal em adulto. Relatos Casos Cir. 2019; 5(3): e2292. [Citado em 20 Mar 2021] Disponível em: https://cdn.publisher.gn1.link/ relatosdocbc.org.br/pdf/v5n3a17.pdf

7. Sala MAS, et col. Má-rotação intestinal associada a obstrução duodenal pela banda de Ladd. Radiol Bras. Jul/Ago 2016; 49(4): 267-276. [Citado em 20 Mar 2021] Disponível em: https://www.scielo.br/pdf/rb/v49n4/ pt_0100-3984-rb-49-04-0271.pdf dores abdominais e vômitos, com potencial evolutivo para isquemia, necrose intestinal e peritonite. Já a forma crônica, possui sintomas inespecíficos, como dor abdominal tipo cólica, intermitente, e vômitos recorrentes. 0 presente caso é caracterizado pela forma aguda, devido volvo de sigmoide, com consequente obstrução intestinal, seguido de dilatação da região cecal e pontos de isquemia.

Assim como a maioria dos casos de evolução rápida ${ }^{1}$, 0 diagnóstico foi estabelecido através de laparotomia exploratória, na qual, observou-se, dentre outras características, cólon descendente, sigmoide e ceco, localizados em região de ângulo hepático e ausência de fixação do ceco em região retroperitoneal. No que tange aos exames de imagens, para Sala et $\mathrm{al}^{7}$, o método de escolha é a Tomografia Computadorizada, realizada neste caso, pois é útil na avaliação das alças e pode auxiliar na avaliação vascular, devido a possível comprometimento. No entanto, outros métodos também podem ser eficazes para o reconhecimento, como o estudo radiológico contrastado, que pode revelar duodeno verticalizado e ausência do ângulo duodenojejunal, em até $80 \%$ dos casos.

Em relação ao manejo em pacientes adultos sintomáticos, não existe padronização quanto à conduta, uma vez que dependerá dos achados intra-operatórios e do tipo de malformação associada. ${ }^{1}$ No caso relatado, em virtude da presença de volvo intestinal e gravidade hemodinâmica, o tratamento envolveu suporte clínico com reanimação volêmica e descompressão gástrica por sonda. Ainda, foi realizada abordagem cirúrgica por laparotomia exploratória de urgência, associado à colectomia e enterectomia dos segmentos isquêmicos mais realização de ileostomia terminal. Segundo Fonseca et $\mathrm{al}^{3}$, mesmo em casos assintomáticos, muitos autores recomendam o tratamento cirúrgico, em razão da imprevisibilidade da ocorrência de volvo e isquemia intestinal. Finalmente, instituída antibioticoterapia de amplo espectro e profilaxia para Tromboembolismo Pulmonar e Trombose Venosa Profunda, associado a cuidados em Unidade de Terapia Intensiva, com alta hospitalar no quinto dia de pós-operatório.

\section{CONCLUSÃO}

É notório que a importância do diagnóstico e diagnóstico diferencial frente ao caso sintomático e até assintomática de má 
rotação intestinal, sendo útil o conhecimento acerca da epidemiologia, sintomatologia meios diagnósticos. Logo o intuito do presente estudo se reforça ao demonstrar possíveis alterações anatômicas intestinais a serem encontradas nesses pacientes, assim como alternativas na conduta cirúrgica com os devidos cuidados necessários.

\section{REFERÊNCIAS}

1. Araújo URMF, et col. Má rotação intestinal em adulto, relato de caso e revisão da literatura. $A B C D$. Arq Bras Cir Dig. 2009; 22(4): 240-242. [Citado em 20 Mar 2021] Disponível em: https://www.scielo. br/pdf/abcd/v22n4/v22n4a13.pdf

2. Cunha FAAA, et col. Má-rotação intestinal: um diagnóstico a ser considerado no abdome agudo em recém-nascidos. Resid Pediatr. 2018; 8(3): 141-146. DOI: 10.25060. [Citado em 20 Mar 2021] Disponível em: http://residenciapediatrica.com.br/detalhes/333/ma-rotacao\%20intestinal-\%20 um\%20diagnostico\%20a\%20ser\%20considerado\%20no\%20abdome\%20a gudo\%20em\%20recemnascidos

3. Fonseca MK, el col. Má rotação intestinal em adulto. Relatos Casos Cir. 2019; 5(3): e2292. [Citado em 20 Mar 2021] Disponível em: https://cdn.publisher.gn1.link/relatosdocbc.org.br/pdf/v5n3a17.pdf

4. Moore KL, Persaud V. Embriologia Básica. 9a edição. Rio de Janeiro: Guanabara. Elsevier. 2004. Capítulo 13. Sistema Digestivo; p. 222-250

5. Neal E, Seymour MD, Dana K, Andersen MD. Laparoscopic Treatment of Intestinal Malrotation in Adults. JSLS. 2005; 9(3): 298 - 301. [Cited on Mar 20 - 2021] Available at: https://www.ncbi.nlm.nih. gov/pmc/articles/PMC3015603/

6. Stephanie A, et col. Intestinal Malrotation - Not Just the Pediatric Surgeon's Problem. J Am Coll Surg. October 2004; 199(4): 628 - 635. [Cited on Mar 20 - 2021] Available at: https://cbc.org.br/wpcontent/uploads/2013/07/032008- JACS2.pdf

7. Sala MAS, et col. Má-rotação intestinal associada a obstrução duodenal pela banda de Ladd. Radiol Bras. Jul/Ago 2016; 49(4): 267-276. [Citado em 20 Mar 2021] Disponível em: https://www.scielo.br/ pdf/rb/v49n4/pt_0100-3984-rb-49-04-0271.pdf

8. Meneghetti GC, et col. Síndrome heterotáxica: relato de caso. São Paulo. Radiol Bras. 2014; 47(1): 54-56. [Citado em 20 Mar 2021] Disponível em: https://www.scielo.br/pdf/rb/v47n1/pt_0100-3984rb-47-01-54.pdf 